\title{
Managing disasters precipitated by natural hazards
}

\author{
V K GAUR \\ CSIR Centre for Mathematical Modelling and Computer Simulation, \\ National Aerospace Laboratories. Belur Campus, Bangalore 560037. \\ India
}

\begin{abstract}
Natural hazards are a piece of the dynamics of the outer working parts of the earth's thermodynamic engines; and their course in many cases cannot be stayed. But disasters caused by them can be significantly minimized by designing and enforcing hazard-resilient land use plans, building codes and other safety and avoidance measures. Current understanding of the space-time characteristics of natural hazards offers considerable insight in designing effective hazard reduction programmes as well as a research agenda that would progressively vitalize this endeavour.
\end{abstract}

Keywords. Natural hazards; disaster reduction.

Life on earth has always been subject to natural catastrophes. Repeatedly, since the dawn of time, sudden or prolonged changes in the supportive environment of the day have wiped out whole species; but evolution proceeded by stimulating the innovative capacities of those surviving, towards the development of evermore viable life-sustaining mechanisms. Human societies too have been hapless victims of recurring scourges wreaked by floods and drought, storms, earthquakes and landslides. The annual toll of life and property exacted by natural hazards has increased rather dramatically since the Industrial Revolution (figure 1). In recent years, for example. between 1964 and 1983.2.5 million people were killed and over 750 million rendered homeless by disasters caused by natural hazards. A single moderate earthquake $(M=6.4)$ that rocked Maharashtra last September, killed over 15.000 people and rendered a large number destitute. Indeed, with the increasing concentration of human populations and industrial activity in super cities (figure 2), the potential threat of natural disasters the world over continues to grow. This threat is particularly high in the third world where 3 factors combine to expose large unwary communities to grave rish.

First. the survival needs of a burgeoning human family drive an increasing number 10 occupy ecological niches of marginal energy, unaware of the slow-developing instabilities that might some day turn into disaster. Second. the one-way environmental degradation caused by the continuing loss of shallow aquifers, forest cover, soils and coastal wetlands. mangrove and coral systems, insidiously erodes the very support hase of the natural systems on which the majority that subsist near the earth are so 


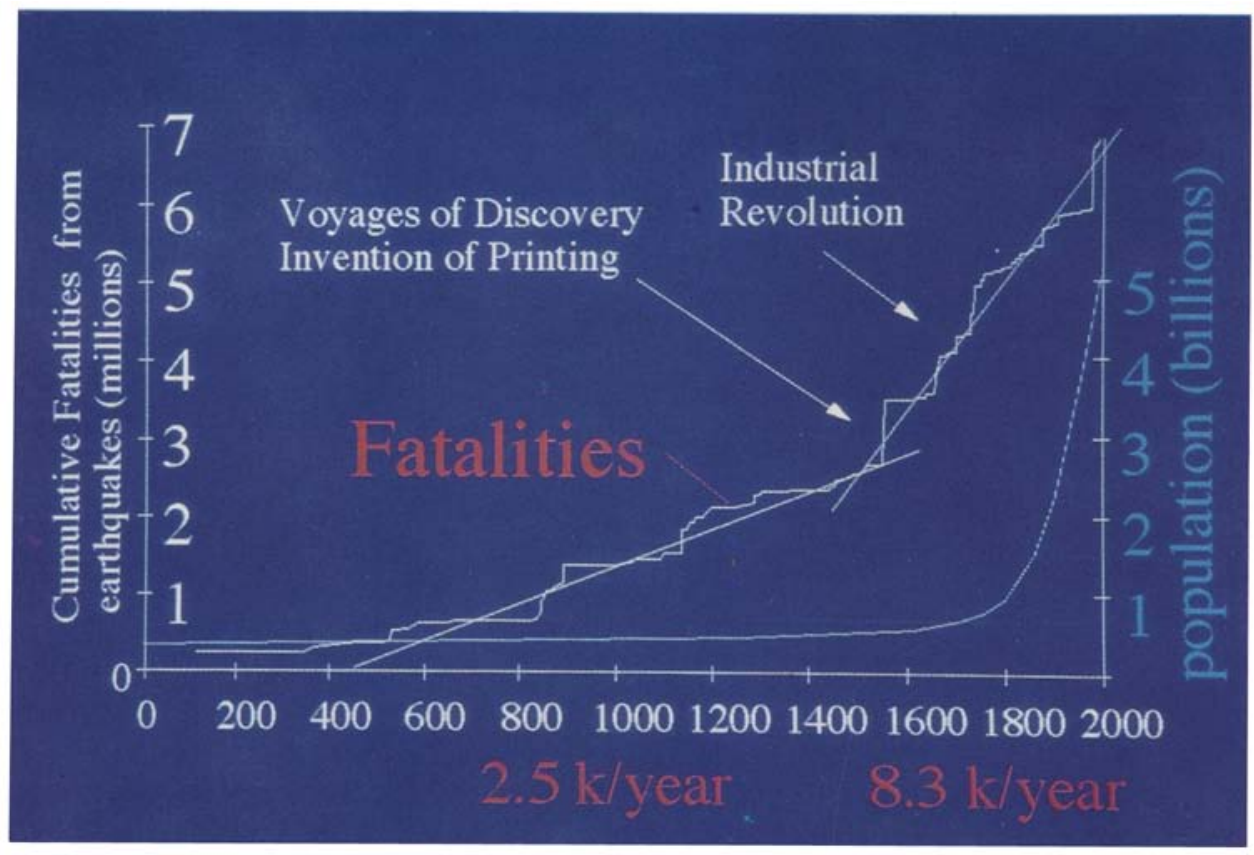

Figure 1. The toll of life exacted by natural hazards.

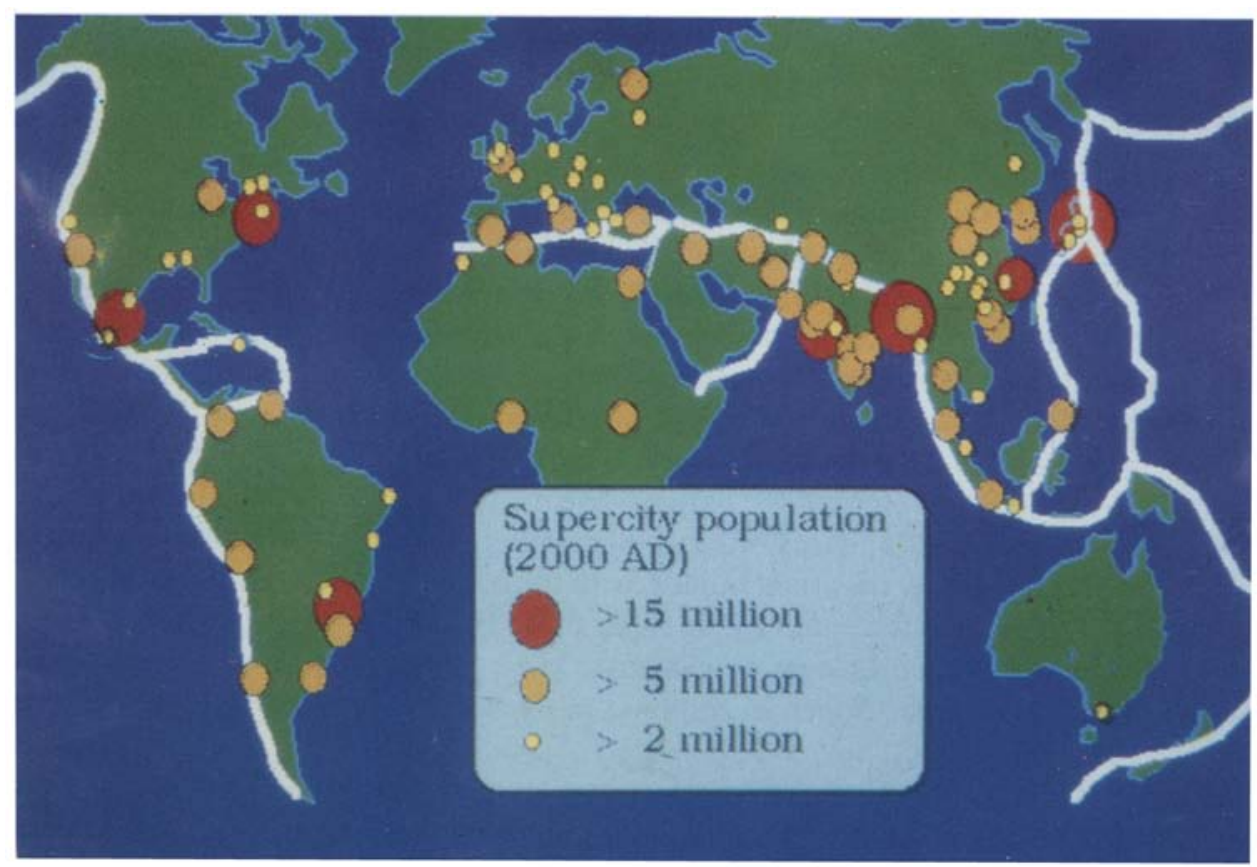

Figure 2. Illustration of the concentration of human population in super cities around the world. 
abjectly dependent. Finally, the poor technological infrastructure for generating hard data on critical earth system parameters, encourages a lackadaisical approach to hazard estimation and design of critical structures and preparedness planning strategies. The latter have the further effect of exacerbating the impact of natural hazards by instilling a sense of false confidence in such measures until, as happens so often, they prove quite inadequate when tragedy strikes.

\section{Natural hazards - the other face of an energetic planet}

Natural hazards result from extreme fluctuations in the dynamic state of our energetic planet. The earth is a vigorously convecting system powered within by radioactivity and primordial heat, and without by the sun's energy which warms it nonuniformly. Its various spheres of solid, liquid and gaseous domains, while subject to their own time scales of processes, thus constantly flow and exchange materials and energy across their multiple interfaces in a ceaseless struggle to attain equilibrium, in turn, fashioning a wondrous variety of ecological systems in passage. However, it is in the very nature of such variously coupled systems that their meta-stable state would periodically excurse to extreme values and even become unpredictably chaotic. Some insights in such processes have been gained in recent years by studying the dynamics of simple coupled systems. Nature, apparently reproduces such behaviour all the time and at various scales. We can visualize these processes at work in the growth of crystals, snow flakes, clouds and drainage channels as well as earthquakes and convective instabilities that breed destructive storms. Natural hazards are thus just the other face of a vibrant youthful planet which alone amongst its four terrestrial sisters organized itself into a system of interactive spheres that would symbiotically regulate its environment for the appearance and sustenance of life forms. Natural hazards are going to be with us forever but disasters need not.

\section{Confronting natural hazards for disaster reduction}

The severity of disasters precipitated by natural hazards is, in fact, determined by our ability or otherwise to confront them with knowledge and commitment. Considerable understanding has now been gained of the evolution and characteristic features of cyclones, floods and drought, earthquakes, volcanic eruptions and landslides. Carefully recorded accounts of past events wherever chronicled, have also led to a recognition of their space-time patterns, while analyses of these patterns have created insights in predicting the probabilities of their occurrences in future. Powerful analytical frameworks and computer simulation models have also developed apace with progress in computational techniques, which enable one to translate these prognostications into quantitative descriptions of probabilistic hazards. These figures when incorporated in the design of land-use patterns, hazard-resistant structures and preparedness strategies, can greatly minimize the impact of Nature's fury. For example, by intelligent management of land slopes backed by scientific mapping and monitoring of the area, Japan has been able to considerably reduce the impact of recurrent landslides from 130,000 dwellings destroyed in 1938 to only 2,000 in 1976. In India too, the existence of a network of coastal radars (figure 5 in Joseph, this volume) and satellite-based surveillance systems to track the evolution of cyclonic storms, enabled the authorities to evacuate over 600,000 people to safe shelters in the wake of the 1990 Andhra cyclone, which attained hurricane intensity. The death toll was thus 
limited to about 1,000 as compared with 20,000 killed by an earlier Andhra cyclone in 1977.

Satellite-based early warning systems in Bangladesh have similarly helped reduce the fatalities caused by cyclones in recent years: from 300,000 deaths in 1970 to 10,000 in 1985.

These few examples of hazard reduction efforts clearly demonstrate how a focussed application of available technologies can significantly reduce the disaster proportions of a natural hazard. The latter examples, of course, relate only to one aspect of hazard reduction strategy, that is, rescue and relief. Yet more remarkable successes can be achieved by advance planning to ensure long-term protection from natural hazards as well as research to gain a clearer definition of the physical processes which will improve our prediction capabilities. This is shown by the Japanese example founded on systematic assessment of landslide hazard, and design and enforcement of wellformulated building and grading codes.

The recognition of these potential capabilities that now lie within our grasp, in enhancing our resilience to face the rigours of natural hazards, led the United Nations to declare the decade of the Nineties as the International Decade for Natural Disaster Reduction (IDNDR). Serious efforts have accordingly been made in some countries, notably in the West and in Japan, for designing a comprehensive hazard reduction programme and its systematic implementation by exploiting the strong commonalities that Disaster Mitigation measures share, irrespective of the nature of specific hazards.

\section{The generic framework for disaster management}

An effective strategy for mitigating disasters caused by natural hazards consists in the design and implementation of four related activities: advance action for long-term protection; preparedness for efficient response to a hazardous event that has happened or is about to happen; recovery and rehabilitation; and research for improving prediction reliability, as well as the design of appropriate engineering and social structures that would effectively embed in the local culture.

\section{Advance planning}

This is the most important activity aimed at providing basic directions for creating an environment for long-term protection and enhancing the resilience of a community in braving natural hazards. The basic grounds prepared in this process also come in handy for implementing rehabilitation measures that would instil confidence in their future viability. This activity involves the following tasks.

* Identification of hazard-prone regions on the basis of historical and current knowledge as well as conceptual anticipations.

* Evaluation of the probability of exceedance of hazard intensities over various intervals in the future, corresponding to the life spans of different types of structures, utilities and systems; and preparation of hazard intensity maps.

* Creation of multihazard Geographical Information Systems and design of land use management schemes.

* Design of engineering specifications for various kinds of structures and systems.

* Assessment of risks faced by existing structures and systems and designs for retrofitting and relocating them wherever necessary. 


\section{Disaster preparedness}

These measures, necessitating horrendous outlays, can obviously be targetted only to specific areas that have already been identified as being vulnerable. They are most effective when well designed protocols for rescue and relief are already in place and can be directed by computer-simulated visualisations of the progress of a suspected or imminent catastrophe. Its basic elements are as follows.

* Design and operational readiness of protocols for effective rescue and relief measures, prevention of cascade disasters such as epidemics, and emergency operation of critical services in the event of their failure.

* Operational prediction models energized by real-time data for forecasting the progressive evolution of a natural hazard, its estimated space-time characteristics and intensity.

* Regular dissemination of information through bulletins carefully designed to evoke a constructive response and avoid panic.

* Rapid response action planning and implementation.

\section{Rehabilitation}

The only way to reduce the continuing impact of natural disasters on affected communities is to provide rapid relief and rehabilitation while at the same time freeing them from the spectre of similar catastrophes in the future. In implementing this activity, therefore, ad hoc measures should be consciously eschewed and strict adherence enforced to follow the recommended land use pattern and codes for engineering design and control.

\section{Research and development}

While all-out efforts should be immediately launched to use globally available knowledge and technologies in estimating and mapping hazard intensities in threatened areas, and in the development of operational prediction systems within this decade, continual improvement of these capabilities will require an equally focussed effort to close the critical gaps in our understanding, through further research. Nature usually offers considerable revealing information in the wake of highly energetic catastrophes, notably earthquake aftershocks and wind fields after the storm landfall. An important research strategy should therefore aim at meticulous scientific preparedness to glean this information contained in the post-disaster behaviour of earth systems. For example, our understanding of earthquake processes in the Himalaya and in the interior of the continent could have progressed by a quantum jump in the last few years, had Indian scientific organizations been ready to monitor details of the immediate aftershock sequences of the recent Bihar (1988), Uttarkashi (1991) and Khillari (1993) earthquakes, and of changes in the attendant strain field.

An illustrative example to draw attention to some of the outstanding problems in hazard reduction that call for continuing research is given in table 1 . Also given are the basic requirements of information products that can be generated by using knowledge already available; and these need to be addressed straightaway. Finally, the last column spells out the prerequisite state-of the-art technology systems needed to realize these goals. These too, should be installed without further delay. 


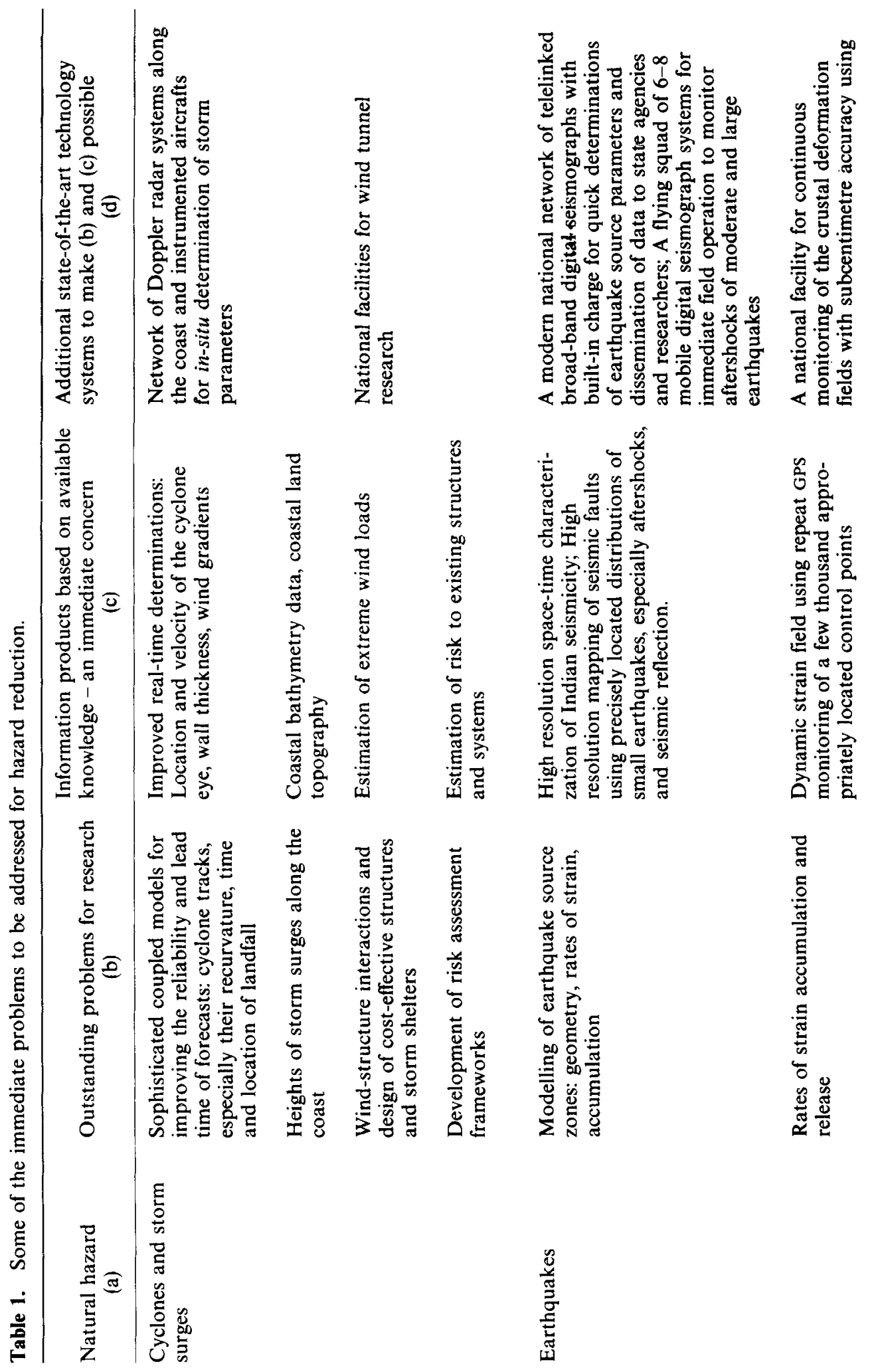



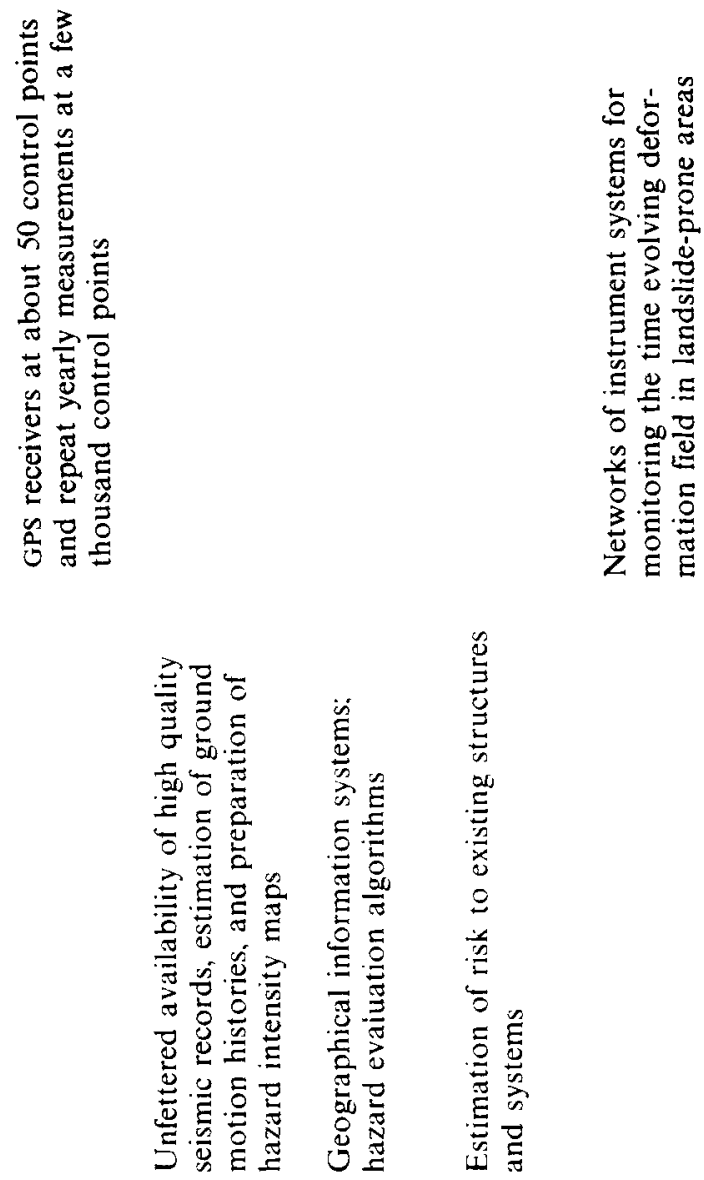

$\stackrel{0}{\Xi}$
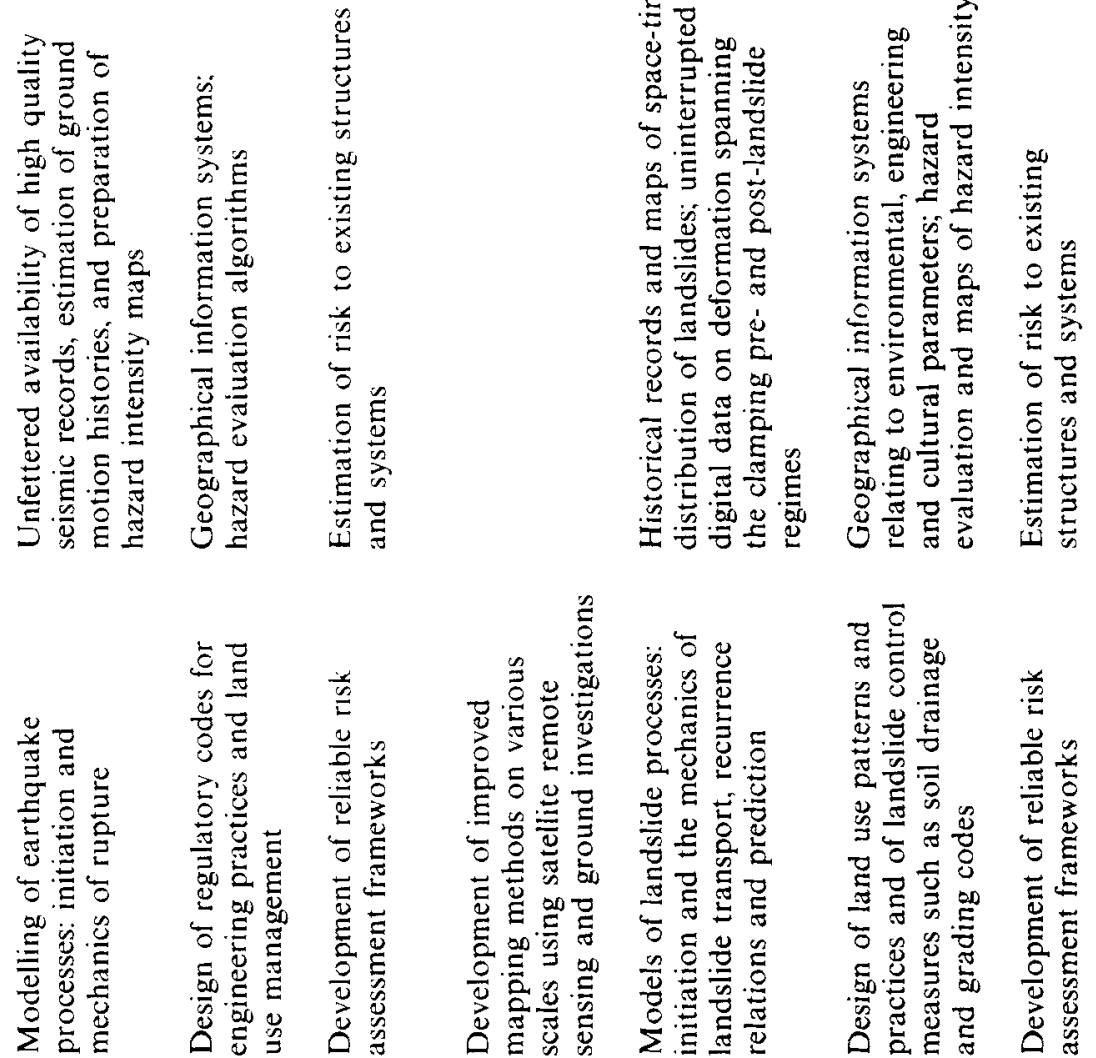
India has taken a number of measures to mitigate the severity of natural disasters caused by floods, storms and drought, but a serious programme to reduce their impact to the levels now possible, through a systematic and focussed application of science and technology, still remains a distant goal. A basic impediment to achieving this goal is the absence of an authentic and reliable information system for environmental data, which forms the core of a disaster-reduction programme.

The extant structure of the government-run scientific organizations charged with the responsibility of data generation, archiving and dissemination, appears to have some inherent difficulties in developing state-of-the-art technology systems capable of generating high quality uninterrupted data sets needed for modern analysis. This fact has to be confronted honestly and squarely whilst establishing the basic information systems. It may even be helpful to seek some insights into the sociology of scientific data-gathering organisations in the country from professionals in the field, so that our next steps in this direction prove to be as vital and flawless as anywhere in the world.

The papers contained in this volume were presented at a short symposium on Disaster Reduction organised at the University of Roorkee as a part of the last annual meeting of the Indian Academy of Sciences. They describe in detail the nature of threats posed by various kinds of natural hazards, and our current endeavours to mitigate their impact. 